\title{
RELACIÓN DE LA BANCA PRIVADA ECUATORIANA EN LA COMERCIALIZACIÓN Y EXPORTACIÓN DEL CAMARÓN PERIODO 2005-2016, APLICACIÓN DE UN MODELO DE VECTORES AUTORREGRESIVOS.
}

\section{RELATION OF THE ECUADORIAN PRIVATE BANKING IN THE COMMERCIALIZATION AND SHRIMP EXPORTATION PERIOD 2005-2016, APPLICATION OF A MODEL OF SELF-REPELLENT VECTORS.}

Francisco Antonio Quinde Rosales, Econ.

Economista Agrícola (Ecuador).

Estudiante de la Maestría en Economía con mención Desarrollo Económico y Políticas Públicas de la Universidad Tecnológica ECOTEC (Ecuador). Asistente de investigación de la Facultad de Economía Agrícola de la Universidad Agraria del Ecuador, Ecuador. fquinde@mgs.ecotec.edu.ec

Víctor Xavier Quinde Rosales, Mgs. Magíster en Economía Agraria (Ecuador). Docente de la Facultad de Economía Agrícola de la Universidad Agraria del Ecuador,

Ecuador. vquinde@uagraria.edu.ec

Gladys Lissette Riquero Malta, Ing. Ingeniera Comercial (Ecuador). Profesional de Control de Energía (CNEL, EP), Ecuador. lissette.riquero@cnel.gob.ec

\section{ARTÍCULO DE INVESTIGACIÓN}

Recibido: 5 de septiembre de 2018.

Aceptado: 20 de noviembre de 2018

\section{RESUMEN}

La presente investigación determina si el crédito otorgado por los Bancos Privados del Ecuador influyó de alguna forma sobre el sector de comercialización y exportación de camarón en el Ecuador para el periodo 2005-2016. Para comprobar dicha relación se realizó una recolección de datos en páginas oficiales como la Súper Intendencia de Bancos, Trade Map 
y Banco Central del Ecuador los cuales sirvieron de base para la realización de varios test econométricos como Modelos De Vectores Autorregresivos, Prueba de Causalidad de Granger, Prueba de Raiz Unitaria, entre otros. Obteniendo como resultado la inexistente relación entre los montos de créditos otorgados y los niveles de exportación para el periodo 2005-2016, esto significa que el papel de la banca dentro del sector exportador de camarón no juega un rol importante sino por el contrario el sector exportador es totalmente independiente con respecto a la banca.

Palabras clave: Crédito, Bancos Privados del Ecuador, Causalidad, Sector exportador de camarón ecuatoriano, Crecimiento económico.

\section{ABSTRACT}

The present investigation determines if the credit granted by the Private Banks of Ecuador influenced in any way With the commercial sector and shrimp exportation in Ecuador for the period 2005-2016. To verify this relationship, a data collection was carried out From oficial web sites such as the Superintendencia de Bancos, Trade Map and Banco Central del Ecuador, which served as the basis for the realization of several econometric tests such as Autoregressive Vector Models, Granger's Causality Test, Unit Root Test, among others. Obtaining as a result the non-existent relationship between the amounts of credits granted and Export levels for the period 2005-2016, this means that the role of the banking within The shrimp sector exportation does not play an important role, however, the export sector it is totally independent with respect to banking.

Keywords: Credit, Private Banks of Ecuador, Causality, Exporting sector of Ecuadorian, Economic growth.

\section{INTRODUCCIÓN}

El presente trabajo aborda el tema de desarrollo económico de una nación, partiendo desde el punto de ser capaz de desarrollar ciertos sectores claves de la economía de un país. El sector camaronero para el Ecuador representa uno de los principales sectores exportadores generando grandes ingresos para el país (Muñoz, Durán \& González, 2017).

Sin embargo, la estructura de los sectores productivos del país carece de la capacidad de auto-inversión necesaria para mantener sus niveles de funcionamiento, es por esta razón que 
la banca juega un papel muy importante dentro de la gran mayoría de los sectores productivos del país mediante la otorgación de créditos.

Por esta razón se planteó un estudio de carácter empírico que demuestre la relación causal que tiene el volumen de créditos otorgados por los Bancos Privados del Ecuador con los volúmenes de exportación de camarón ecuatoriano mediante la elaboración de distintos modelos econométricos.

Se obtuvo como resultado que la Banca privada ecuatoriana no tiene un papel importante dentro del sector exportador de camarón, ya que no existe relación alguna entre las variables planteadas, es por esta razón que se recomienda realizar futuras investigaciones con diferentes variables que sean capaces de influir en los niveles de camarón exportado, esto con el fin de identificarlos y estimularlos para impulsar aún más a este sector tan rentable para la economía ecuatoriana.

\section{REVISIÓN TEÓRICA}

El crecimiento económico dentro de la Economía de una nación es uno de los principales objetivos a alcanzar, puesto que tal crecimiento nos da herramientas y medios para mejorar de cierta forma las condiciones de vida de los ciudadanos. Es por esta razón que este concepto se vuelve tan importante, ya que sin crecimiento económico no es posible obtener un desarrollo económico.

Adam Smith, en su obra maestra titulada "An Inquiry into the Nature and Causes of the Wealth of Nations" en el año de 1776, señaló que el crecimiento de una nación dependerá de la sinergia de ciertos elementos fundamentales como son: la acumulación de capital, estructuras productivas, división del trabajo y regulación estatal mas no intervención; es decir, Smith no contemplaba la idea de un mercado sin participación estatal sino, por el contrario, que este sea capaz de regular el mercado mediante instituciones que funcionen de forma adecuada, la llamada "mano Invisible".

Smith (1776) señala,

Lo que ocasiona una subida en los salarios no es el tamaño efectivo de la riqueza nacional sino su permanente crecimiento. Los salarios, por lo tanto, no son más altos en los países más ricos sino en los que prosperan más, o en los que se hacen ricos más rápidamente (p.67). 
Lo anterior se convirtió en el pensamiento central de la escuela clásica, sin embargo, con el pasar de los años esta teoría evolucionó en un nuevo pensamiento denominado Neoclásico. Ros (2013), define el pensamiento neoclásico de crecimiento como,

...enfoques se centran en el lado de la oferta de la economía, en el sentido de que el nivel de producto y su tasa de crecimiento están restringidos por la acumulación de factores y el progreso tecno-lógico exógeno, los efectos de productividad de la acumulación de capital en presencia de rendimientos crecientes a escala, o bien los factores del lado de la oferta que afectan la innovación y el progre-so tecnológico ( $p$. 17).

Keynes en su obra titulada "Teoría General del Empleo, el Interés y el Dinero" en 1936, explica que el desarrollo económico se puede lograr a través de la correcta distribución del ingreso, una adecuada tasa de interés, estimulando el gasto público y generando un aumento de la demanda agregada lo que se traduce en un incremento del ingreso nacional (Keynes, 1936).

Petit (2013), señala que

Keynes acabó con la idea de que una economía de mercado conduce automáticamente al pleno empleo. Esa pérdida de fe en los automatismos reguladores de la economía abrió la puerta a la necesidad de la política económica, esto es, de la intervención del Estado en la economía para alcanzar una situación de pleno empleo (p. 5).

Solow explica como el crecimiento económico de los Estados Unidos no es producto de la acumulación de capital, ya que esta no garantiza el crecimiento económico, por el contrario, la inversión en nueva tecnología y mano de obra más calificada garantiza el crecimiento de un determinado sector o país (Oriol, 2006).

Otros pensadores como Shumpeter consideran que el desarrollo económico es producto de la innovación y del trabajo armónico entre fuerzas materiales e inmateriales, las primeras están compuestas por los factores productivos clásicos, es decir, tierra, capital y trabajo. Mientras que las fuerzas inmateriales están representadas por aquellas posiciones sociales o hechos técnicos. Shumpeter explica que solo se puede lograr un desarrollo económico si estos factores se unen y trabajan de forma dinámica entre ellos logrando el desarrollo siempre que exista una innovación relevante (Montoya, 2004).

Todas estas teorías nos dan una idea claro de lo que es el crecimiento económico y lo importante que es acumular el capital, pero a su vez generar un incremento continuo de este a través de mejorar los procesos productivos, siendo estos capaces de dinamizar la economía 
de una nación buscando siempre el bienestar de su población. Buendia (2013) señala que "La competencia es una parte esencial para el desarrollo y la mejora constante de nuestras habilidades, en ausencia de competidores resulta difícil determinar si una persona, empresa o nación es o no eficiente" (p.14). Esto explica a breves rasgos lo importante que resulta dentro del crecimiento económico la competencia y eficiencia.

En el caso ecuatoriano los sectores económicos que sustentan el crecimiento económico de la nación aún carecen de sostenibilidad, ya que sus procesos en su gran mayoría son precarios, es por esta razón que se asume que el crédito dinamiza la producción de todos los sectores. En síntesis, se asume la idea que solo el financiamiento de la banca para el caso del Ecuador, garantiza una producción continua y estable debido a las condiciones del sector agrícola ecuatoriano.

De igual forma es importante destacar como investigaciones empíricas permiten a la academia realizar estudios ajustados a la realidad a partir de la interpretación personal de distintas variables, de esta manera se puede analizar aspectos como el crecimiento económico de forma experimental y sobre todo más ajustadas a la realidad de la sociedad.

Chystilin (2011), explica empíricamente en una ecuación de desarrollo, como los diferentes factores económicos, tales como PIB y Población, afectan el crecimiento económico permitiendo a partir de este modelo establecer predicciones y cuantificar los posibles impactos al variar el volumen de los factores. Marroquín y Ríos (2012), realizan un análisis de carácter empírico en el cual demuestran mediante las variables Investigación y Desarrollo los distintos niveles de crecimiento económico al establecer una función capaz de explicar el impacto de estas sobre el aumento de la riqueza.

Such, Zapata, Risso, Brida y Pereyra (2009), analizan el crecimiento económico como resultado del estímulo del turismo en Colombia realizando un modelo econométrico capaz de explicar la relación entre las variables PIB Per cápita y Gasto Real en Turismo. Garcia (2001), realizó un estudio de carácter empírico sobre el crecimiento económico, determinando que las naciones en vías de desarrollo deben su crecimiento a cambios estructurales en sus cadenas productivas.

\section{MATERIALES Y MÉTODOS}

El presente artículo se enmarca dentro del método empírico, inductivo y deductivo. Los datos utilizados en el análisis en cuestión fueron extraídos de páginas oficiales de instituciones como la Súper Intendencia de Bancos y Trade Map, siendo estos datos la base para la realización 
de varias pruebas econométricas en el sistema Eviews 10, las cuales analizaron la relación entre el crédito otorgado por los Bancos Privados del Ecuador y las exportaciones del camarón ecuatoriano en toneladas métricas para el periodo 2005-2016.

Se propone un análisis capaz de medir la estacionalidad de las variables mediante el uso de la prueba Dickey-Fuller Aumentada. Stock et al. (2012) menciona que el contraste DFA para una raíz unitaria autorregresiva contrasta la hipótesis nula $\mathrm{H} 0: \delta=0$ frente a la hipótesis alternativa $\mathrm{H} 1: \delta<0$ en la siguiente regresión:

$$
\Delta y_{t}=\beta_{1}+\beta_{2} t+\delta y_{t-1} \sum_{i=1}^{m} \alpha i \Delta y_{t-1}+\varepsilon_{t}
$$

Entendiendo que:

H0: La variable es no estacionaria; tiene raíz unitaria

H1: La variable es estacionaria; no tiene raíz unitaria

Para establecer la relación se usó el Modelo de Vectores Autoregresivos (VAR), dicho modelo argumenta la existencia de simultaneidad entre el conjunto de variables a tratarse en igualdad de condiciones sin distinción de variables exógenas ni endógenas. Se denomina vector autorregresivo ante la existencia de un valor rezagado de la variable dependiente y la existencia de un vector de dos o más variables.

El modelo VAR toma en cuenta las siguientes ecuaciones:

$$
\begin{aligned}
& M_{1 t}=\alpha+\sum_{j=1}^{k} \beta_{j} M_{t-j}+\sum_{j=1}^{k} Y_{j} R_{t-j}+\mu_{1 t} \\
& R_{t}=\alpha^{\prime}+\sum_{j=1}^{k} \theta_{j} M_{t-j}+\sum_{j=1}^{k} Y_{j} R_{t-j}+\mu_{2 t}
\end{aligned}
$$

Se plantean las siguientes hipótesis:

Hipótesis 1: La relación existente entre las variables es igual a un año siendo considerada de corto plazo.

Hipótesis 2: La relación existente entre las variables es mayor a un año siendo considerada de largo plazo. 
A su vez se realizó la prueba de Granger con el fin de interpretar la causalidad que genera una variable sobre otra, ya sea en términos de $X$ a $Y$ o de $Y$ a $X$. Según el test de Granger plantea las siguientes hipótesis.

Hipótesis 1. Causalidad unidireccional de Crédito a Toneladas; rechazo de la hipótesis 1. Hipótesis 2. Causalidad unidireccional de Toneladas a Crédito; rechazo de la hipótesis 2. Hipótesis 3. Causalidad bidireccional entre las variables; ambas hipótesis son rechazadas.

Hipótesis 4. Variables independientes; ambas hipótesis no son rechazadas.

Para probar las hipótesis se toma en cuenta la probabilidad de Fischer (Prob). Si el valor p se encuentra en el valor mínimo de rechazo, menor al 5\% ó 0,05 se rechaza una de las hipótesis.

\section{RESULTADOS}

Dentro del periodo estudiado, 2005-2016, las instituciones financieras privadas asignaron en el Ecuador \$732'092.421,97 en créditos hacia las actividades de comercio al por mayor de camarón congelado, otros procesos y exportación. Cerrando en el cuarto semestre del año 2016 con una tasa de crecimiento del $29 \%$ con respecto al primer trimestre del 2005, con una tasa trimestral promedio de crecimiento para el periodo estudiado del $50 \%$.

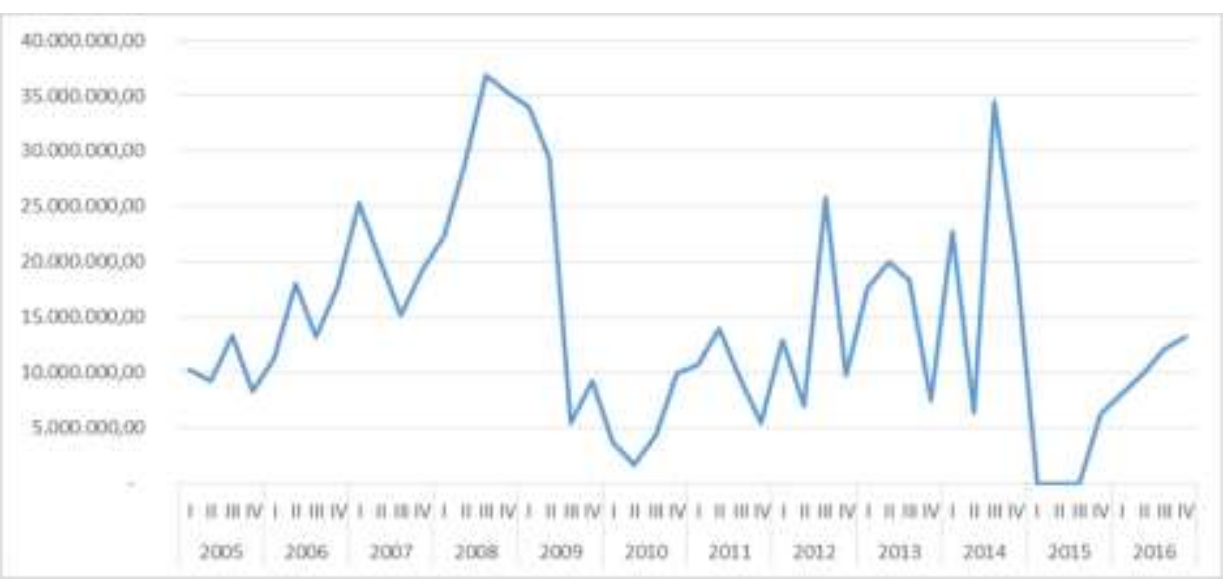

Figura 1. Evolución del volumen de crédito otorgado por bancos privados (2005-2016). Fuente: Súper Intendencia de Bancos (2016).

La provincia que se benefició en mayor proporción de los créditos otorgados dentro del periodo estudiado, fue la provincia del Guayas con el $81 \%$ en promedio para dicho periodo, mientras que EI Oro alcanzó el 7\% de participación y el restante 12\% se divide en las demás provincias. 
Finalmente se destaca la participación del Banco del Pichincha quien impulso en este periodo a dichas actividades con la mayor cantidad de asignaciones $\$ 255$ '260.625,00 representando esto el $43 \%$ del total del monto asignado por todas las instituciones para dichas actividades.

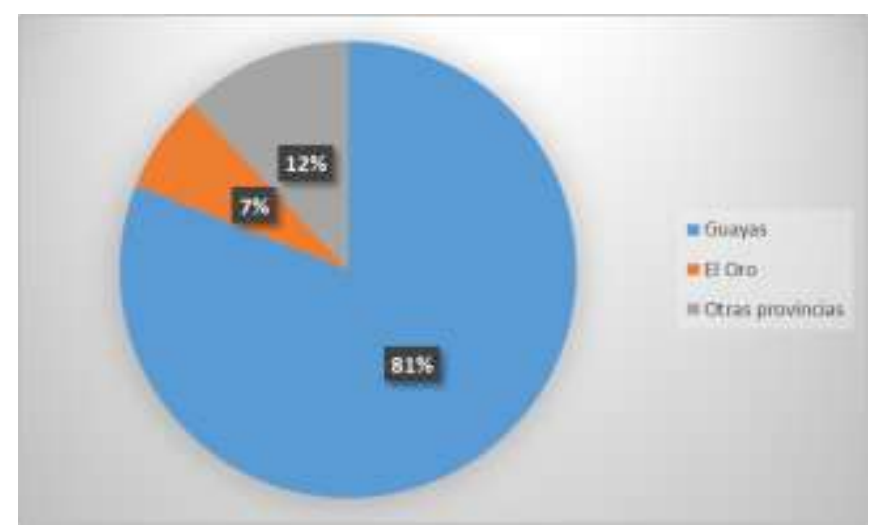

Figura 2. Participación por provincia dentro del volumen de créditos asignados (2005-2016). Fuente: Súper Intendencia de Bancos (2016).

El volumen de exportaciones del camarón ecuatoriano hacia el resto del mundo para el periodo estudiado ascendió a \$12'880.764,00 (miles), aumentando en un 512\% para el cuarto trimestre del 2016 con respecto al primer trimestre del año 2005 a una tasa promedio de crecimiento para dicho periodo del $176 \%$ con respecto al primer trimestre. Por su parte el volumen de exportaciones en toneladas de camarón se incrementó en un 334\% para el último semestre del 2016 con respecto al primer semestre del año 2005, a una tasa promedio de crecimiento con respecto al primer trimestre del 131\% representando todo esto 2'096.095 de toneladas exportadas.

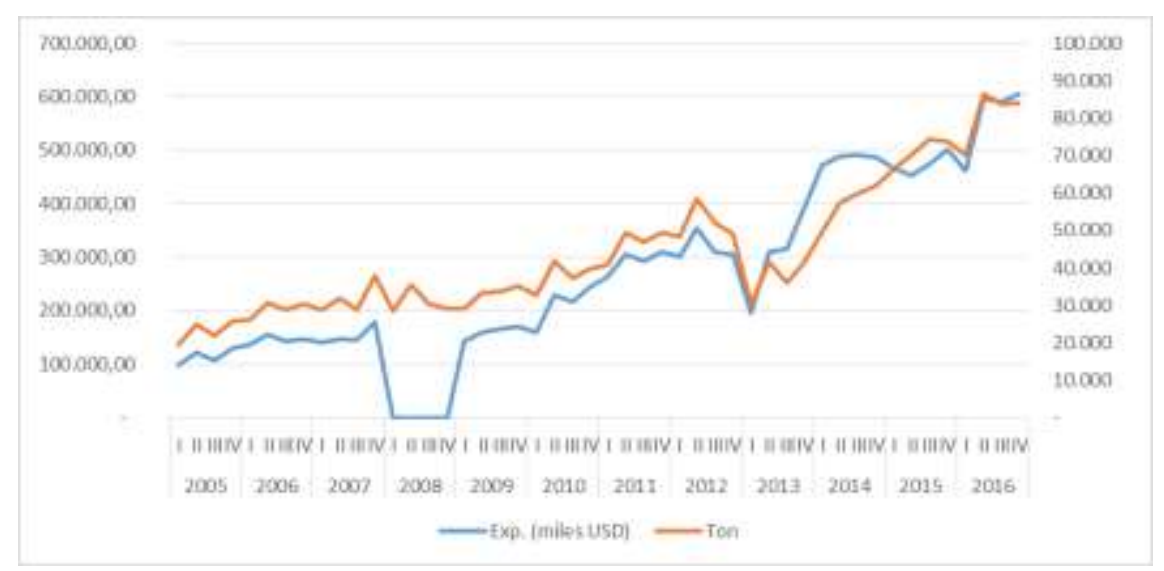

Figura 3. Exportaciones de camarón ecuatoriano (2005-2016).

Fuente: Trade Map. 
Se utilizó la prueba de Dickey Fuller que nos muestra la tendencia determinística o estocástica de las variables, los resultados obtenidos nos muestran que la variable Toneladas Métricas (TM) posee raíz unitaria mientras que la variable Créditos no posee raíz unitaria, siendo estas no estacionarias y estacionarias respectivamente.

Null Hypothesis: TM has a unit root

Exogenous: Constant

Lag Length: 1 (Automatic - based on SIC, maxlag=9)

\begin{tabular}{lll}
\hline \hline & t-Statistic & Prob. $^{*}$ \\
\hline \hline Augmented Dickey-Fuller test statistic & 0.536314 & \multirow{2}{*}{0.9862} \\
\hline Test critical values: $1 \%$ level & -3.581152 & \\
$5 \%$ level & -2.926622 & \\
$10 \%$ level & -2.601424 & \\
& & \\
\hline \hline
\end{tabular}

*MacKinnon (1996) one-sided p-values.

Figura 4 a. Prueba de Raíz Unitaria Toneladas métricas.

Fuente: Elaboración propia.

.Null Hypothesis: CREDITOS_USD_has a unit root Exogenous: Constant

Lag Length: 2 (Automatic - based on SIC, maxlag=9)

\begin{tabular}{llll}
\hline \hline & & t-Statistic & Prob.* \\
\hline \hline Augmented Dickey-Fuller test statistic & -3.138269 & 0.0307 \\
\hline Test critical values: & 1\% level & -3.584743 & \\
& $5 \%$ level & -2.928142 & \\
& $10 \%$ level & -2.602225 & \\
& & \\
\hline \hline
\end{tabular}

*MacKinnon (1996) one-sided p-values.

Tabla 1. Prueba de Raíz Unitaria Créditos.

Fuente: Elaboración propia. 
Al establecer la longitud máxima de rezagos sobre la cual se determine la relación entre las variables estudiadas se obtiene el vector autorregresivo óptimo, para ello se utilizó la prueba de razón de verosimilitud, los criterios de Error de Predicción Final y los estadísticos de Akaike, Schwarz y Hannan-Quinn, estos criterios presentan un desacuerdo entre el rezago 2 y 3.

VAR Lag Order Selection Criteria

Endogenous variables: TM

CREDITOS_USD_

Exogenous variables: $\mathrm{C}$

Date: 09/04/18 Time: 15:15

Sample: 2005Q1 2016Q4

Included observations: 38

\begin{tabular}{|c|c|c|c|c|c|c|}
\hline Lag & LogL & LR & FPE & AIC & SC & $H Q$ \\
\hline 0 & -1089.155 & NA & $2.99 e+22$ & 57.42923 & $\begin{array}{r}57.51542 \\
55.73164\end{array}$ & 57.45989 \\
\hline 1 & -1047.988 & $\begin{array}{r}75.83376 \\
10.30915\end{array}$ & $4.24 \mathrm{e}+21$ & 55.47308 & * & $\begin{array}{r}55.56507 \\
55.52453\end{array}$ \\
\hline 2 & -1042.053 & * & $\begin{array}{c}3.84 \mathrm{e}+21 \\
3.64 \mathrm{e}+21\end{array}$ & $\begin{array}{r}55.37120 \\
55.31369\end{array}$ & 55.80215 & * \\
\hline 3 & -1036.960 & 8.309215 & * & * & 55.91701 & 55.52835 \\
\hline 4 & -1034.419 & 3.879037 & $3.97 e+21$ & 55.39046 & 56.16616 & 55.66644 \\
\hline 5 & -1033.829 & 0.837876 & $4.83 e+21$ & 55.56995 & 56.51803 & 55.90727 \\
\hline 6 & -1033.163 & 0.876297 & $5.89 e+21$ & 55.74542 & 56.86588 & 56.14407 \\
\hline 7 & -1030.407 & 3.336894 & $6.50 \mathrm{e}+21$ & 55.81087 & 57.10370 & 56.27085 \\
\hline 8 & -1028.713 & 1.871683 & $7.68 e+21$ & 55.93227 & 57.39748 & 56.45358 \\
\hline 9 & -1026.609 & 2.103585 & $9.02 e+21$ & 56.03208 & 57.66966 & 56.61472 \\
\hline 10 & -1021.967 & 4.153842 & $9.45 e+21$ & 55.99826 & 57.80822 & 56.64223 \\
\hline
\end{tabular}

* indicates lag order selected by the criterion

LR: sequential modified LR test statistic (each test at $5 \%$ level)

FPE: Final prediction error

AIC: Akaike information criterion

SC: Schwarz information criterion 
HQ: Hannan-Quinn information criterion

Tabla 2. Criterios de Selección del Orden de Retrasos.

Fuente: Elaboración propia.

Para resolver dicha discrepancia se elaboró un test de residuos, el cual estima el comportamiento de estos dentro de los intervalos de confianza, obteniendo como resultado que el modelo con 2 rezagos es el óptimo (Figura 4 y 5).

Autocorrelations with Approximate 2 Std.Err. Bounds

$\operatorname{Cor}(\mathrm{TM}, \mathrm{TM}(-\mathrm{i}))$

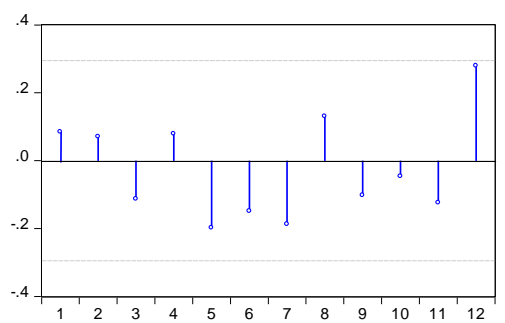

Cor(CREDITOS_USD_,TM(-i))

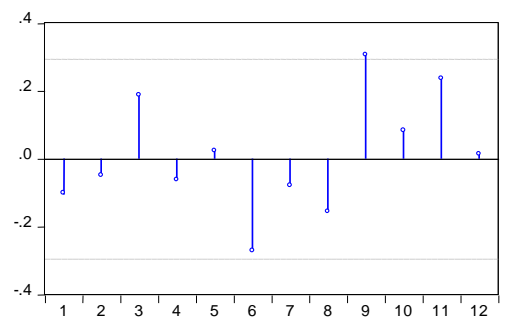

Cor(TM,CREDITOS_USD_(-i))

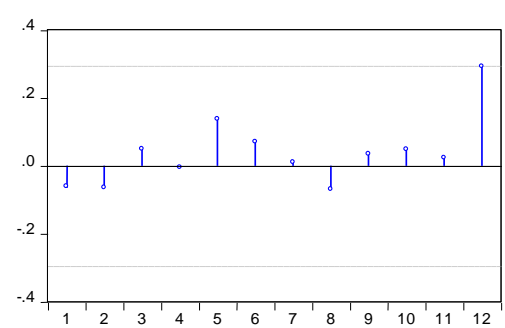

Cor(CREDITOS_USD_CREDITOS_USD_(-i))

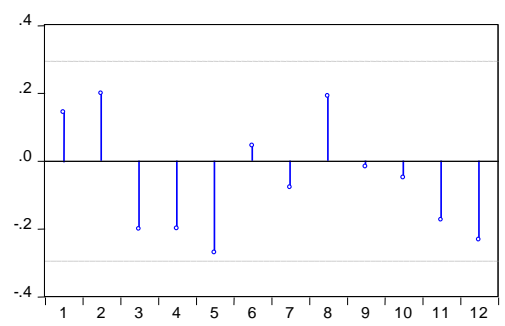

Figura 4. Test de residuos- rezago 2.

Fuente: Elaboración propia. 
Autocorrelations with Approximate 2 Std.Err. Bounds

$\operatorname{Cor}(\mathrm{TM}, \mathrm{TM}(-\mathrm{i}))$

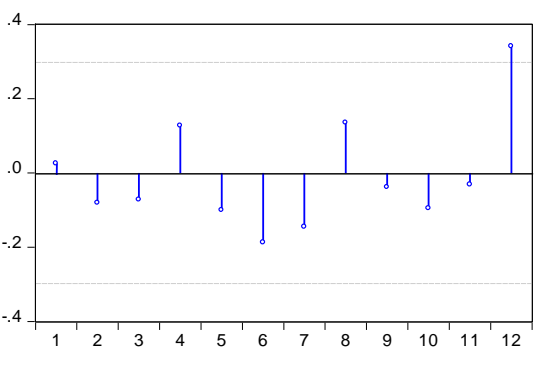

Cor(CREDITOS_USD_TM(-i))

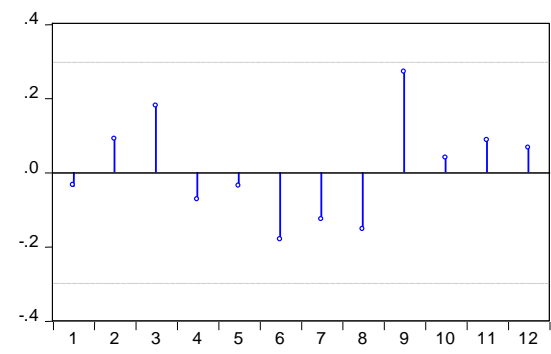

Cor(TM,CREDITOS_USD_(-i))

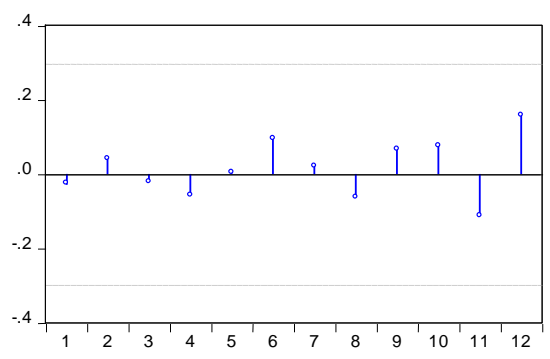

Cor(CREDITOS_USD_CREDITOS_USD_(-i))

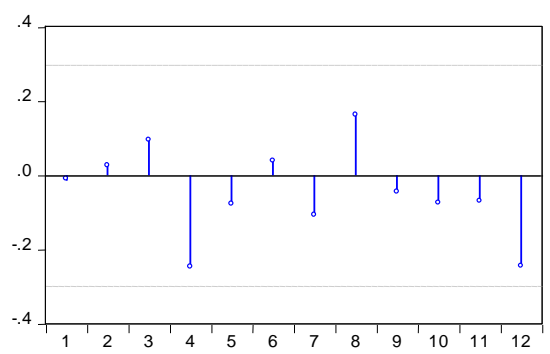

Figura 5. Test de residuos- rezago 3.

Fuente: Elaboración propia.

De esta forma se establece el modelo óptimo para 3 rezagos, observándose los coeficientes relevantes como R-Squared el cual tiene un nivel de cobertura del $90 \%$ y F-Statistic igualmente elevado. Esto significa que el modelo es confiable (Tabla 3).

Vector Autoregression Estimates

Date: 09/04/18 Time: 15:18

Sample (adjusted): 2005Q3 2016Q4

Included observations: 46 after adjustments

Standard errors in ( ) \& t-statistics in [ ]

\begin{tabular}{lcc}
\hline \hline & & \multicolumn{1}{c}{$\begin{array}{c}\text { CREDITOS_ } \\
\text { USD_ }\end{array}$} \\
\hline \hline $\mathrm{TM}(-1)$ & 0.596714 & 12.33823 \\
& $(0.14235)$ & $(204.092)$ \\
& {$[4.19179]$} & {$[0.06045]$} \\
& & \\
$\mathrm{TM}(-2)$ & 0.422423 & -65.58338 \\
& $(0.14789)$ & $(212.034)$ \\
& {$[2.85629]$} & {$[-0.30931]$}
\end{tabular}


CREDITOS_USD_(-

1) $\begin{array}{rr}5.01 \mathrm{E}-06 & 0.294610 \\ (0.00010) & (0.14693) \\ {[0.04888]} & {[2.00513]}\end{array}$

CREDITOS_USD_(-

\begin{tabular}{lcc} 
2) & $-7.25 \mathrm{E}-05$ & 0.348732 \\
& $(0.00010)$ & $(0.14549)$ \\
& {$[-0.71449]$} & {$[2.39695]$} \\
C & & \\
& 2070.989 & 7842241. \\
& $(3272.27)$ & $(4691472)$ \\
& {$[0.63289]$} & {$[1.67159]$} \\
\hline \hline R-squared & 0.904961 & 0.342769 \\
Adj. R-squared & 0.895689 & 0.278649 \\
Sum sq. resids & $1.32 \mathrm{E}+09$ & $2.70 \mathrm{E}+15$ \\
S.E. equation & 5664.372 & 8121036. \\
F-statistic & 97.60086 & 5.345739 \\
Log likelihood & -460.1543 & -794.4831 \\
Akaike AlC & 20.22410 & 34.76014 \\
Schwarz SC & 20.42287 & 34.95890 \\
Mean dependent & 45230.25 & 15490417 \\
S.D. dependent & 17538.29 & 9561767.
\end{tabular}

Determinant resid covariance (dof adj.)

2.10E+21

Determinant resid covariance

$1.67 \mathrm{E}+21$

Log likelihood

$-1254.446$

Akaike information criterion

54.97592

Schwarz criterion

55.37345

Number of coefficients

10

Tabla 3. Modelo propuesto- Rezago 2.

Fuente: Elaboración propia. 
Con el fin de establecer la causalidad, se elaboró la prueba de Causalidad de Granger obteniendo como resultado una inexistente causalidad entre las variables estudiadas en cualquier sentido (Tabla 4).

VAR Granger Causality/Block Exogeneity Wald Tests

Date: 09/04/18 Time: 15:21

Sample: 2005Q1 2016Q4

Included observations: 46

Dependent variable: TM

\begin{tabular}{llll}
\hline \hline Excluded & Chi-sq & df & Prob. \\
\hline \hline CREDITOS_USD_ & 0.612610 & 2 & 0.7362 \\
\hline \hline All & 0.612610 & 2 & 0.7362 \\
\hline \hline
\end{tabular}

Dependent variable: CREDITOS_USD_

\begin{tabular}{llll}
\hline \hline Excluded & Chi-sq & df & Prob. \\
\hline \hline TM & 0.481680 & 2 & 0.7860 \\
\hline \hline All & 0.481680 & 2 & 0.7860 \\
\hline \hline
\end{tabular}

Tabla 4. Prueba de Causalidad de Granger.

Fuente: Elaboración propia.

Finalmente se recreó la tabla resumen de cointegración de Johansen con el fin de observar si existe algún modelo que cointegre las variables en el largo plazo, obteniendo como resultados la inexistencia de cointegración a largo plazo (Tabla 5.). 
Date: 09/04/18 Time: 15:21

Sample: 2005Q1 2016Q4

Included observations: 45

Series: TM CREDITOS_USD_

Lags interval: 1 to 2

Selected

(0.05 level $\left.{ }^{\star}\right)$

Number of

Cointegrati

ng

Relations

by Model

\begin{tabular}{|c|c|c|c|c|c|}
\hline \multicolumn{2}{|c|}{ Data Trend:None } & None & Linear & Linear & Quadratic \\
\hline \multirow[t]{2}{*}{ Test Type } & \multicolumn{2}{|c|}{ No InterceptIntercept } & Intercept & Intercept & Intercept \\
\hline & No Trend & No Trend & No Trend & Trend & Trend \\
\hline Trace & 0 & 0 & 0 & 0 & 0 \\
\hline Max-Eig & 0 & 0 & 0 & 0 & 0 \\
\hline
\end{tabular}

${ }^{*}$ Critical values based on MacKinnon-Haug-Michelis (1999)

Tabla 5. Test de Cointegración de Johansen.

Fuente: Elaboración propia.

\section{CONCLUSIONES}

Los resultados obtenidos muestran el evidente crecimiento de los créditos otorgados por los Bancos Privados del Ecuador, hacia actividades relacionadas con la comercialización y exportación de camarón, sin embargo, si estas son comparadas con los volúmenes de exportación en toneladas métricas existe una marcada diferencia a favor de las toneladas exportadas en el periodo 2005-2016 con 305 puntos de diferencia en cuanto a su tasa de crecimiento durante dicho periodo.

El modelo econométrico que se utilizó nos muestra la inexistente relación entre las variables Créditos y Toneladas métricas, esto significa que no existe relación alguna entre dichas variables ya que ninguna de ellas es capaz de explicar el comportamiento de la otra. 
Finalmente, se concluye que el crédito otorgado por los Bancos Privados del Ecuador hacia el sector de la comercialización y exportación de camarón, no es de vital importancia ya que no existe relación entre estas variables, se recomienda realizar estudios que exploren variables distintas con el fin de determinar factores que influyan de forma directa a este sector de la economía logrando así impulsarlos y dinamizar dicho sector.

\section{REFERENCIAS BIBLIOGRÁFICAS}

Buendía, E. A. (2013). El papel de la Ventaja Competitiva en el desarrollo económico de los países. Análisis Económico, vol. XXVIII, núm. 69, 14.

Chystilin, D. (2011). Estudio sobre desarrollo económico: principios básicos, modelo y evidencia empírica . Ensayos Revista de Economía-Volumen XXX, No. 1.

Garcia, B. (2001). Educación, capital humano y crecimiento. Ciencia Ergo Sum, vol. 8, núm. 1.

Hidalgo, C. A. (1998). El pensamiento económico sobre el Desarrollo. De los Mercantilistas al PNUD.

Keynes, J. M. (1936). Teoría general del empleo, el interés y el dinero. Palgrave Macmillan.

Marroquìn, J., \& Rios, H. (2012). Inversión en investigación y crecimiento económico: un análisis empírico desde la perspectiva de los modelos de I+D. Investigación Económica, vol. LXXI, núm. 282.

Muñoz, S., Durán, G., \& González, I. (2017). ANÁLISIS DEL SECTOR CAMARONERO ECUATORIANO Y SUS VENTAJAS COMPETITIVAS Y COMPARATIVAS PARA ENCARAR UN MERCADO INTERNACIONAL COMPETITIVO. Revista Cumbres.

Petit, J. G. (2013). La teoría económica del desarrollo desde Keynes hasta el nuevo modelo neoclásico del crecimiento. Revista Venezolana de Análisis de Coyuntura, vol. XIX, núm. 1.

Ricoy, C. J. (2005). La teoría del crecimiento económico de Adam Smith. Economía y Desarrollo, vol. 138, núm. 1.

Ros, J. (2013). Introducción a "Repensar el desarrollo económico, el crecimiento y las instituciones". Economíaunam, vol. 10, núm. 30.

Smith, A. (1776). An Inquiry into the Nature and Causes of the Wealth of Nations. 
Such, M. J., Zapata, S., Risso, W. A., \& Brida, J. G. (2009). Turismo y crecimiento económico: Un análisis empírico de Colombia. Estudios y Perspectivas en Turismo, vol. 18, núm. 1.

Wooldridge, J. M. (2009). Introducción a la Econometría. Un enfoque moderno. 